Rev. Int. Contam. Ambie. 35 (Residuos solidos en México) 7-9, 2019

DOI: 10.20937/RICA.2019.35.esp02.01

\title{
MANEJO DE RESIDUOS SÓLIDOS EN MÉXICO
}

\author{
Sara OJEDA-BENITEZ ${ }^{1,3 *}$ y Claudia E. SALDAÑA DURÁN ${ }^{2,3}$
}

\footnotetext{
${ }^{1}$ Instituto de Ingeniería, Universidad Autónoma de Baja California, Calle de la Normal s/n, Insurgentes Este, Mexicali, Baja California, México, c.p. 21280

${ }^{2}$ Universidad Autónoma de Nayarit, Ciudad de la Cultura Amado Nervo, Tepic, Nayarit, C.P. 631901

${ }^{3}$ Sociedad Mexicana de Ciencia y Tecnología Aplicada a Residuos Sólidos, A.C., Priv. Molcajete 44, Fraccionamiento Hacienda de las Fuentes, Calimaya, Estado de México, Mexico, C.P. 52227

*Autora de correspondencia: sara.ojeda.benitez@uabc.edu.mx
}

\section{EDITORIAL}

En México, como en otros países, el manejo de residuos sólidos es tema que requiere atención, como uno de los retos ambientales para ser atendido e insertado en la agenda pública y política. Es importante destacar que el manejo de residuos no sólo involucra los efectos ambientales y de salud pública, también implica el uso de recursos naturales.

En 2015, de acuerdo con el Banco Mundial, en México la generación de residuos fue de $53.1 \mathrm{mi}-$ llones de toneladas, la generación per cápita diaria alcanzó 1.2 kilogramos en promedio (Kaza et al. 2018). De los residuos que se generan, más del 50 por ciento son orgánicos, una gran parte de los cuales se origina a partir de la pérdida y el desperdicio de alimentos; el primero ocurre durante la producción, almacenamiento y distribución de productos alimenticios; el segundo, cuando los alimentos consumidos se desechan. De las muchas consecuencias ambientales asociadas a esta situación está la contribución a las emisiones de gases de efecto invernadero (FAO 2013). En México, las estimaciones indican que para 2020, el sector de residuos sólidos será la quinta fuente de emisiones de gases de efecto invernadero en el país (SEMARNAT 2016).

Por lo que se debe promover su gestión integral, para reducir su generación y valorizarlos antes de la disposición final; estas prácticas influirán en la reducción de extracción de recursos, de energía y agua que se utilizan en los procesos productivos, así como en la emisión de gases de efecto invernadero, obteniendo con ello beneficios económicos, sociales y ambientales.
Por ello en este número especial sobre manejo de residuos sólidos en México, se presentan resultados de algunos casos de estudio que se realizaron en ciudades mexicanas, en los que se analiza la gestión de los residuos sólidos, se evalúan sistemas de manejo de residuos sólidos urbanos (RSU), se aborda el problema del manejo de medicamentos veterinarios caducos, se aplican los principios de ecología industrial para valorizar los residuos de la agroindustria, se presenta el uso de herramientas como los sistemas de información geográfica para la optimización del transporte de residuos y la identificación de sitios de disposición final, se propone un modelo para el biosecado de residuos orgánicos y se evalúan los efectos de la composición, la biodegradabilidad y las condiciones de confinamiento sobre la biodegradación de los RSU en un sitio de disposición final.

El primer artículo, analiza la alianza de los grupos de interés, entre ellos el gobierno, la sociedad y la academia, desde la perspectiva de la gestión de los residuos sólidos. Los resultados indican que se puede llegar a establecer una gestión integral de RSU con la participación de los diferentes actores que conforman los grupos de interés, se deben fortalecer los vínculos de cooperación y participación entre las partes involucradas, así como establecer políticas orientadas a la gestión integral de los RSU que consideren los diversos factores que influyen en la toma de decisiones.

El segundo artículo, analiza las prácticas de manejo de RSU en los cuatro municipios centrales de la Zona Metropolitana de Guadalajara (ZMG): 
Guadalajara, Zapopan, Tlaquepaque y Tonalá. Presenta un análisis del marco normativo, los programas y políticas públicas, así como los discursos en relación con el manejo de los RSU en México, el autor menciona que éstos han evolucionado sustancialmente en las últimas dos décadas en contraste con las prácticas de consumo y disposición de $\mathrm{RSU}$, así como las estrategias de manejo que no han evolucionado al mismo ritmo para ser congruentes con los ideales propuestos en la normatividad; propone cinco indicadores para evaluar el avance en el manejo de RSU con un enfoque sustentable, en el periodo analizado, los cuales pueden ser aplicados en otros municipios.

El siguiente artículo aborda el problema de los medicamentos veterinarios caducos. Los autores señalan que aunque en México existen mecanismos para la correcta gestión ambiental de residuos farmacéuticos, la ausencia de una normatividad específica para medicamentos veterinarios caducos hace que los métodos de manejo, tratamiento y disposición final sean inadecuados. Uno de los problemas que identifican es que la normatividad y la legislación ambiental mexicanas no son claras en cuanto al manejo, tratamiento y disposición final de preparaciones farmacéuticas de tipo veterinario ya que se centran en los medicamentos de uso humano. Idealmente los medicamentos caducos y sobrantes de medicamentos deberían ser puestos a disposición de empresas especializadas en el manejo y disposición final de los mismos. Lo cual se cumple, en cierta medida, para los medicamentos de uso humano, para los que la normatividad es clara, pero no para los de uso veterinario, para los cuales es ambigua. Esta falta de claridad propicia que los encargados de establecimientos veterinarios adopten diversas formas de desechar los medicamentos caducos y sobrantes, al no conocer la forma más adecuada, de tal manera que en uno de cada cinco establecimientos veterinarios los residuos de medicamentos siguen siendo desechados por el drenaje o junto con sus residuos sólidos urbanos, por lo que su disposición final es el relleno sanitario y pueden en ambos casos alcanzar los mantos freáticos.

En el cuarto artículo se analiza la problemática asociada a la valorización de residuos en el sector productivo agroindustrial de Guanajuato. Proponen alternativas de valorización de la corriente de residuos de este sector, aplicando la ecología industrial, para establecer alternativas cerrando ciclos de materia y aprovechamiento de energía en sistemas industriales. Se presenta una propuesta de valorización de los residuos no aprovechados usando los flujos de residuos de unas empresas como materias primas para otras empresas. Se propone establecer planes de manejo de residuos especiales, aplicando principios de ecología industrial, con lo cual se pueden reducir los residuos generados por el sector agroindustrial y al mismo tiempo aprovechar residuos generados por empresas de giros diferentes al porcino, lo que conllevaría un beneficio ambiental y un beneficio económico al ejecutar esos planes de manejo.

El quinto artículo, aborda el problema que enfrentan los municipios en el transporte de RSU a sitios de disposición final, el cual está asociado al gasto de recursos económicos y emisiones atmosféricas derivadas del traslado de los residuos a su disposición final. Los autores aplican los sistemas de información geográfica (SIG) como herramienta para la optimización de rutas de transporte, tomando como caso de estudio la Ciudad de México. Para ello crearon bases de datos georreferenciadas con información de las estaciones de transferencia (ET) y de los sitios de disposición final (SDF) a los cuales se envían los RSU generados. Destacan que la optimización de las rutas de transporte de RSU es indispensable para evitar la generación de gastos innecesarios. Con los resultados de su trabajo muestran que el análisis de redes en SIG es una herramienta útil para el cálculo de rutas y estimación de distancias entre puntos de una red de caminos. Entre los hallazgos mencionan algunos de los problemas que deben ser atendidos, como el poner atención en las distancias que se están recorriendo para llevar los residuos sólidos desde las ET de la ciudad a los SDF foráneos, las cuales son muy largas, por lo que una de las primeras prioridades para el gobierno de la Ciudad de México debería ser el reordenamiento de las rutas ET-SDF con el fin de reducir costos de traslado y hacer el transporte más eficiente. Además deben buscar alternativas de SDF porque en algunos casos se están depositando en lugares que no fueron diseñados para recibir grandes cantidades de residuos, lo cual es una medida insostenible.

En el sexto artículo los autores identifican zonas potenciales para la ubicación de un relleno sanitario de residuos sólidos en el municipio de Tepic. Para ello se realizó un análisis espacial del municipio contrastando cuatro criterios señalados en la Norma Oficial Mexicana NOM-083-SEMARNAT-2003, distancia mínima a centros de población, infraestructura (vías de comunicación área), cauces y cuerpos de agua, y áreas naturales protegidas, además dos rasgos naturales, pendiente y cobertura, así como uso del terreno. La inclusión de estos rasgos limita las áreas con potencial, al evitar aquellas susceptibles de des- 
lizamientos de tierra y afectaciones a la vegetación natural, como consecuencia de las obras de ingeniería y operación asociadas al relleno sanitario.

En el séptimo articulo se desarrolla un modelo matemático determinístico para representar el proceso de biosecado de una pila de residuos sólidos orgánicos dentro de una estructura tipo invernadero. El modelo incluye transferencia de masa, transferencia de calor, crecimiento microbiano y degradación de la materia orgánica. La formulación matemática está integrada por un sistema de cuatro ecuaciones diferenciales obtenidas mediante balances de masa y energía en el centro de la pila: evaporación de agua, crecimiento microbiano, consumo de materia orgánica, y temperatura. Las limitaciones que tiene el modelo es que no predice la temperatura y humedad en la superficie de la pila ni en las capas intermedias y tampoco predice el comportamiento de dichos parámetros durante el volteo.

El último artículo, presenta un estudio en el que se evaluaron los efectos de la composición, la biodegradabilidad y las condiciones de confinamiento sobre la biodegradación de los RSU confinados en un sitio de disposición final. La biodegradabilidad se evaluó mediante la caracterización de compuestos lignocelulósicos, la biodegradación por sólidos volátiles (SV) y las condiciones de confinamiento por la temperatura, $\mathrm{pH}$ y humedad. Los resultados muestran que los contenidos de lignina (LI) y de celulosa influyen en una baja biodegradabilidad de la fracción orgánica $(\mathrm{FO})$.

\section{REFERENCIAS}

FAO (2013) Food Wastage Footprint - Impacts on Natural Resources. Organización de las Naciones Unidas para la Alimentación y la Agricultura [en línea]. http://www. fao.org/3/i3347e/i3347e.pdf 3/05/2019

Kaza S., Yao L. C., Bhada-Tata P. y Van Woerden F. (2018). What a Waste 2.0: A Global Snapshot of Solid Waste Management to 2050 World Bank Publications. Washington, EUA, 272 pp.

DOI: 10.1596/978-1-4648-1329-0

SEMARNAT (2016) Informe de la situación del medio ambiente en México. Compendio de Estadísticas Ambientales. Indicadores Clave, de Desempeño Ambiental y de Crecimiento Verde 2015 Secretaría de Medio Ambiente y Recursos Naturales, México [en línea]. https://www.gob.mx/cms/uploads/attachment/ file/161446/Cap_CC_completo.pdf 03/05/2019 